\title{
Correspondence \\ Forgetting fear associations through tES: which memory process might be critical?
}

Translational Psychiatry (2017) 7, e1046; doi:10.1038/tp.2017.26; published online 28 February 2017

We read with interest the article by Abend and colleagues ${ }^{1}$ titled 'Modulation of fear extinction processes using transcranial electrical stimulation', which adds relevant insights to the extremely limited literature on the effect of non-invasive brain stimulation on fear extinction mechanisms. Given the interest in using brain stimulation for treatment of anxiety disorders and post-traumatic stress disorder, research examining the impact of non-invasive brain stimulation on mechanisms involved in the maintenance and treatment of these disorders is critical.

In their study, Abend and colleagues examined the effects of two different types of non-invasive transcranial electrical stimulation (tES; that is, direct current (DC) and alternating current (AC) stimulation) on fear extinction processes in healthy humans to assess a potential relevance/application of these protocols in therapeutic context. Thus, the authors decided to test three separate groups of participants (that is, DC, AC and Sham stimulation) by targeting the medial prefrontal cortex, which is known to mediate fear extinction mechanisms (for example, Milad and Quirk ${ }^{2}$ ), in a 3-day protocol. On the basis of previous research, they expected that excitability-enhancing anodal tDCS over the ventromedial prefrontal cortex would improve extinction, most probably via activation of GABAergic neurons of the amygdala, ${ }^{3}$ and that low-frequency tACS during extinction might reduce fear memory consolidation via long-term depression-like effects. Skin conductance and self-report responses were examined to assess the effect of the provided treatments in fear extinction.

The results are not in line with the predicted outcomes as AC stimulation enhanced the expression of fear responses following extinction, whereas DC stimulation led to overgeneralization of the fear response to non-reinforced stimuli for the skin conductance parameter, compared to the sham stimulation.

The authors provide different, not mutually exclusive, explanations for interpreting the reported results (for example, the temporal momentum associated to the tES delivery and/or the involvement of other brain regions than the mesial prefrontal cortex and/or brain circuits). All these aspects make good sense, given the fact that the dorsal anterior cingulate cortex, which is situated nearby the mesial prefrontal cortex, has antagonistic effects on extinction, ${ }^{4}$ and that extinction might be timingspecific. These aspects call for spatially and temporally more specific stimulation protocols.

The application of tES for 20 min, during the fear extinctionlearning phase, as it was done in that study, raises more issues that might add further insights to the current discussion on these unexpected results. The process of fear association learning, as well as any learning process, consists of two distinct mechanisms, namely encoding and consolidation. According to definitions of eminent scholars in the field, the encoding process refers to 'operations that take place when the individual originally experiences an event initiate the storage of information representing the event in episodic memory ${ }_{i}^{\prime 5}$ the consolidation process refers to 'the idea that neural processes transpiring after the initial

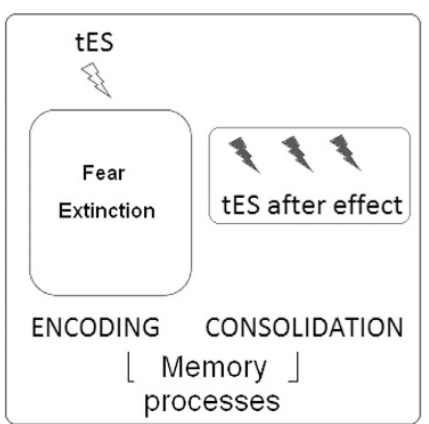

Figure 1. Diagram of transcranial electrical stimulation (tES) effects on memory processes (that is, encoding and consolidation) during and after the extinction phase.

registration of information contribute to the permanent storage of memory'. ${ }^{6}$

The research design proposed by Abend and colleagues does not allow to discern whether the reported results (i) are due to a modulation of the encoding process, which takes place during the fear extinction phase; (ii) reflect a tES after-effect phenomenon, that might last for some hours (for example, refs 7-9), and therefore reflect the influence of $\mathrm{tES}$ on the consolidation process; (iii) reflect the influence of tES on both processes (see Figure 1 for a general schema).

The application of $\mathrm{tES}$ in a research design that separates the 'encoding' from the 'consolidation' process might provide important insights for the current discussion, as distinct mechanisms (for example, Cho et al. $^{10}$ ) for these two processes have been described with regard to extinction of fear memories. At the same time, it would provide highly relevant insights for the application of tES in the clinical field, as it might clarify which process should be targeted by electrical stimulation to empower its effect of a memory extinction therapy.

\section{CONFLICT OF INTEREST}

The authors declare no conflict of interest.

CM Vicario ${ }^{1,2}$, MA Nitsche ${ }^{3,4}$ and K Felmingham ${ }^{1}$

${ }^{1}$ School of Psychology, University of Tasmania, Hobart, TAS,

Australia;

${ }^{2}$ Wolfson Centre for Clinical and Cognitive Neuroscience, School of Psychology, Bangor University, Bangor, UK;

${ }^{3}$ Leibniz Research Centre for Working Environment and Human Factors, Dortmund, Germany and

${ }^{4}$ University Medical Hospital Bergmannsheil, Bochum, Germany E-mail: carmelo.vicario@utas.edu.au

\section{REFERENCES}

1 Abend R, Jalon I, Gurevitch G, Sar-El R, Shechner T, Pine DS et al. Modulation of fear extinction processes using transcranial electrical stimulation. Transl Psychiatry 2016; 6: e913. 
2 Milad MR, Quirk GJ. Neurons in medial prefrontal cortex signal memory for fear extinction. Nature 2002; 420: 70-74.

3 Amano T, Unal CT, Pare D. Synaptic correlates of fear extinction in the amygdala. Nat Neurosci 2010; 13: 489-494.

4 Likhtik E, Pelletier JG, Paz R, Paré D. Prefrontal control of the amygdala. J Neurosci 2005; 25: 7429-7437.

5 Tulving E, Kapur S, Craik Fl, Moscovitch M, Houle S. Hemispheric encoding/ retrieval asymmetry in episodic memory: positron emission tomography findings. Proc Natl Acad Sci USA 1994; 91: 2016-2020, Review.

6 Nadel L, Moscovitch M. Memory consolidation, retrograde amnesia and the hippocampal complex. Curr Opin Neurobiol 1997; 7: 217-227, Review.

7 Nitsche MA, Nitsche MS, Klein CC, Tergau F, Rothwell JC, Paulus W. Level of action of cathodal DC polarisation induced inhibition of the human motor cortex. Clin Neurophysiol 2003; 114: 600-604.

8 Nitsche MA, Paulus W. Sustained excitability elevations induced by transcranial DC motor cortex stimulation in humans. Neurology 2001; 57: 1899-1901.
9 Batsikadze G, Moliadze V, Paulus W, Kuo MF, Nitsche MA. Partially non-linear stimulation intensity-dependent effects of direct current stimulation on motor cortex excitability in humans. J Physiol 2013; 591: 1987-2000.

$10 \mathrm{Cho} \mathrm{JH}$, Deisseroth K, Bolshakov VY. Synaptic encoding of fear extinction in mPFCamygdala circuits. Neuron 2013; 80: 1491-1507.

\section{cc) (i)}

This work is licensed under a Creative Commons Attribution 4.0 International License. The images or other third party material in this article are included in the article's Creative Commons license, unless indicated otherwise in the credit line; if the material is not included under the Creative Commons license, users will need to obtain permission from the license holder to reproduce the material. To view a copy of this license, visit http://creativecommons.org/licenses/ by/4.0/

(c) The Author(s) 2017 